\title{
Quasi-single-sideband radio over fiber transmission with a polymer-based waveguide microring resonator
}

\author{
Xiuyou Han \\ Jianing Zhang \\ Dalian University of Technology \\ School of Physics and Optoelectronic \\ Engineering \\ Photonics Research Center \\ Dalian, 116024, China \\ E-mail: xyhan@dlut.edu.cn

\section{Linghua Wang} \\ Dalian University of Technology \\ School of Physics and Optoelectronic \\ Engineering \\ Photonics Research Center \\ Dalian, 116024, China \\ and \\ Ghent University-IMEC \\ INTEC-Department \\ Photonics Research Group \\ Ghent B-9000, Belgium

\section{Yiying Gu} \\ Meng Wang \\ Dalian University of Technology \\ School of Physics and Optoelectronic \\ Engineering \\ Photonics Research Center \\ Dalian, 116024, China
}

\section{Jie Teng}

Dalian University of Technology

School of Physics and Optoelectronic Engineering

Photonics Research Center

Dalian, 116024, China

and

Ghent University-IMEC

INTEC-Department

Photonics Research Group

Ghent B-9000, Belgium

\section{Jinyan Wang \\ Xigao Jian}

Dalian University of Technology

School of Chemical Engineering

Dalian 116024, China

\section{Geert Morthier}

Ghent University-IMEC

INTEC-Department

Photonics Research Group

Ghent B-9000, Belgium

\author{
Mingshan Zhao \\ Dalian University of Technology \\ School of Physics and Optoelectronic \\ Engineering \\ Photonics Research Center \\ Dalian, 116024, China
}

\begin{abstract}
Integrated waveguide microwave photonic filters (MPFs) have the potential to bring down volume, weight, and power consumption of signal processing equipment besides the common advantages of discrete-component-based MPFs. A polysiloxane-liquid polymer-based optical waveguide microring resonator was designed and fabricated by a simple ultraviolet-based soft-imprint technology, with which the quasi-single-sideband filtering for the 10 to $22 \mathrm{GHz}$ microwave signal was realized and $20 \mathrm{Mbps}$ quadrature phase shift keying signal carried by $14.35 \mathrm{GHz}$ microwave transmission over a $25 \mathrm{~km}$ single mode fiber was demonstrated. 2011 Society of Photo-Optical Instrumentation Engineers (SPIE) [DOI: 10.1117/1.3657818]
\end{abstract}

Subject terms: polymer waveguide; microring resonator; microwave photonic filter; radio over fiber.

Paper 110999R received Aug. 23, 2011; revised manuscript received Oct. 10, 2011; accepted for publication Oct. 13, 2011; published online Nov. 16, 2011.

\section{Introduction}

Various microwave photonic filters (MPFs) have been extensively studied in view of their promising applications to broadband wireless access networks, radio over fiber systems, radars for moving target identification, satellite communication systems, and so forth. ${ }^{1}$ These filters leverage the advantages of photonic signal processing by moving the filtering operation to the optical domain, which offers the benefits of wide bandwidth, low loss, and natural immunity to electromagnetic interference. ${ }^{2,3}$

Generally speaking, the majority of MPFs have been implemented with commercially available discrete components such as single mode fibers (SMFs), couplers, fiber Bragg gratings, and one or more optical sources. ${ }^{1,2}$ In contrast to discrete components, integrated waveguide MPFs have the potential to bring down volume, weight, and power consumption of signal processing equipments, ${ }^{4}$ which is essentially important in aircraft and space satellite communication systems, ${ }^{5-7}$ especially if integration with electro-optical modulators (EOMs) and photodetectors (PDs) is possible.

Polymer is a promising material of choice for photonic integrated waveguide devices because, when synthesized and processed properly, it offers high performance in terms of low-loss, smaller birefringence, high tunability in terms of large thermo-optic coefficient, environmentally stable, high yields, and low cost. ${ }^{8}$ Moreover, it provides an ideal integration platform where foreign material systems such as yttrium 


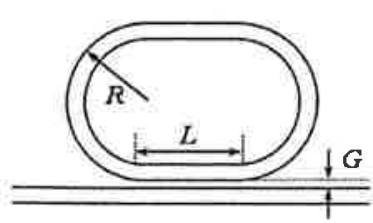

(a)

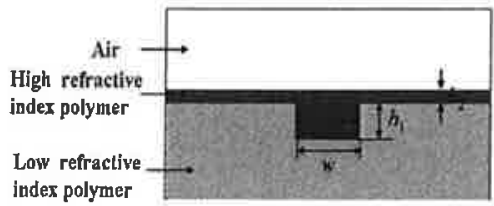

(b)

Fig. 1 (a) Schematic of microring resonator and (b) cross section of polymer waveguide. iron garnet, lithium niobate, and semiconductor devices (such as lasers, detectors, and amplifiers) can be inserted into an etched groove in a planar lightwave circuit to enable full function modules on a single substrate. ${ }^{9.10}$ Polymer-based integrated waveguide devices for microwave photonic signal processing, such as the compact optical true time delay lines, ${ }^{11,12}$ high bandwidth EOMs ${ }^{13}$ and reconfigurable bandpass filters, ${ }^{14}$ have been demonstrated.

In this paper, a polysiloxane-liquid (PSQ-L) polymerbased microring resonator was designed and fabricated by a simple ultraviolet (UV)-based soft-imprint technology, with which the quasi-single-sideband (SSB) filtering response was realized. The chromatic dispersion induced fading effect was suppressed effectively and a $20 \mathrm{Mbps}$ quadrature phase shift keying (QPSK) signal carried by $14.35 \mathrm{GHz}$ microwave was transmitted successfully over $25 \mathrm{~km} \mathrm{SMF}$.

\section{Polymer-Based Waveguide Microring Resonator}

With the polymeric material PSQ-L developed in our group, ${ }^{15,16}$ an all-pass type single microring resonator [Fig. 1(a)] with an inverted ridge waveguide cross section [Fig. 1(b)] as a notch filter was designed and fabricated by a UV-based soft-imprint technology.

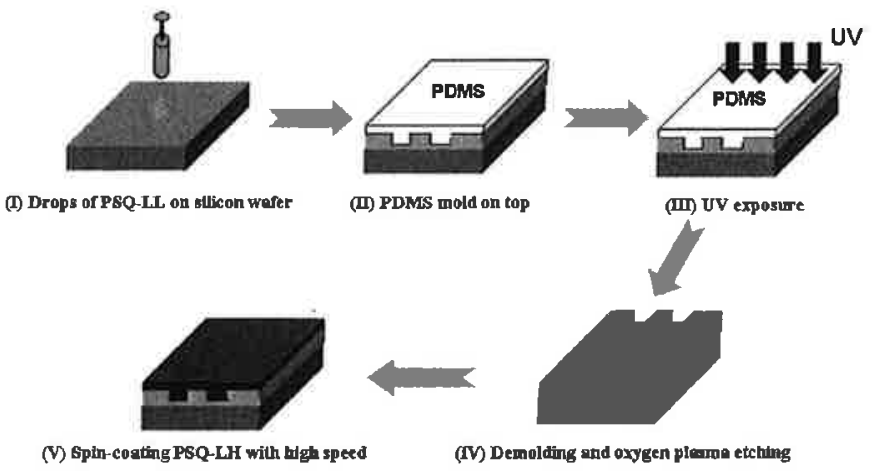

(a)

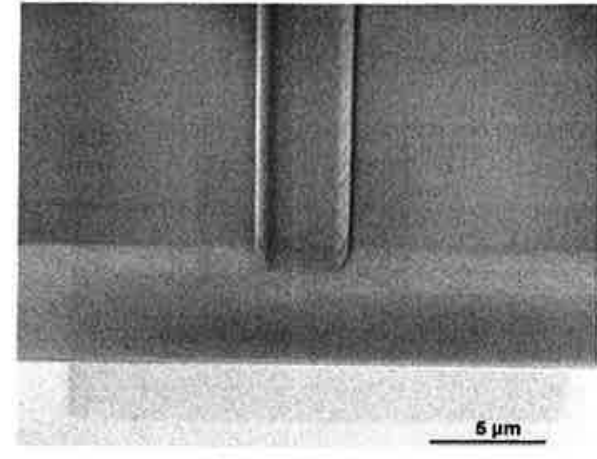

(b)

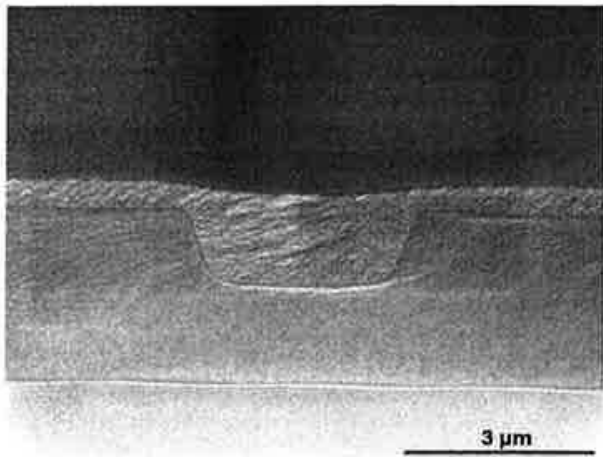

(c)

Fig. 2 (a) UV-based soft-imprint flow, (b) SEM picture of imprinted under-cladding trench, and (c) waveguide cross section after spin-coating the high refractive index layer. 


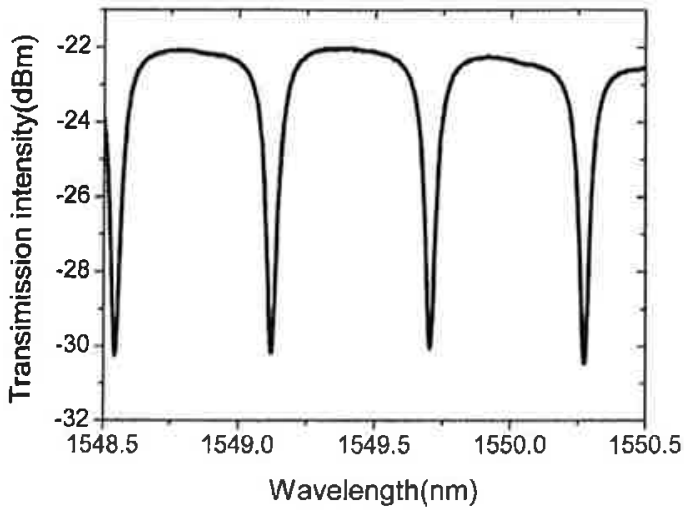

(a)

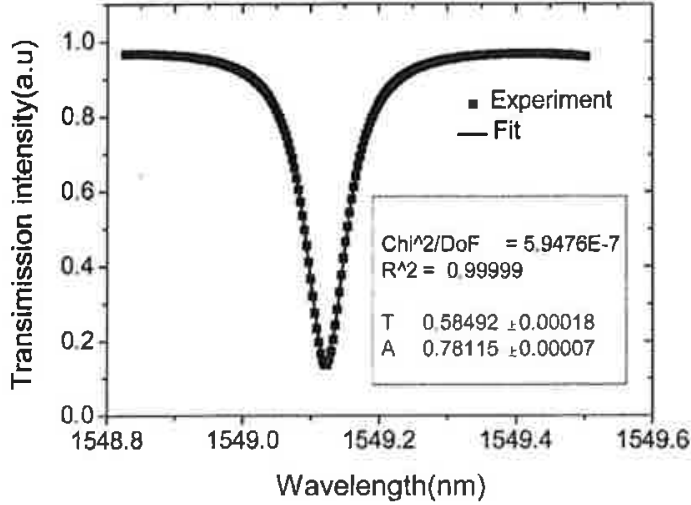

(b)

Fig. 3 (a) The measured optical transmission spectrum of the polymer-based microring resonator (TE mode) and (b) the parameters of the microring resonator through fitting the experimental data.

The normalized transmission function of the microring resonator can be expressed as ${ }^{17}$

$$
I(\lambda)=\frac{T-2 \sqrt{T} \sqrt{A} \cos \left[\frac{2 \pi}{\lambda} \cdot n_{e f f}(2 \pi R+2 L)\right]+A}{1-2 \sqrt{T} \sqrt{A} \cos \left[\frac{2 \pi}{\lambda} \cdot n_{e f f}(2 \pi R+2 L)\right]+T A},
$$

where $R$ is the radius of the ring; $L$ is the straight waveguide length of the directional coupler; $A$ is the power attenuation coefficient per round-trip; $T$ is the power transmission coefficient of the directional coupler; $n_{\text {eff }}$ is the effective index of the waveguide; $\lambda$ is the wavelength of optical wave in vacuum. The ring is on resonance when $n_{\mathrm{eff}}(2 \pi R+2 L)=m \lambda_{m}$, where $m$ is an integer. Equation (1) shows that the resonant wavelength $\lambda_{m}$ is eliminated from the output of the microring and the maximal extinction ratio (ER) occurs when the critical coupling condition $(T=A)$ is satisfied. ${ }^{17}$ With the refractive indexes of core (PSQ-LH, $n_{1}=1.52$ ) and cladding (PSQ-LL, $n_{2}=1.45$ ) waveguides, the designed structural parameters of the microring resonator (shown in Fig. 1) are as follows: $R=400 \mu \mathrm{m}, L=150 \mu \mathrm{m}, G=1 \mu \mathrm{m}, w=3 \mu \mathrm{m}$, $h_{1}=2 \mu \mathrm{m}$ and $h_{2}=0.8 \mu \mathrm{m}$, which fulfill the single-mode waveguide condition and negligible bending loss.

The simple UV-based soft-imprint technology as shown in Fig. 2(a) is used for the fabrication of the waveguide microring resonator. Unlike in conventional imprint processes, the imprint step for structuring is done first on the cladding layer rather than on the core layer and is followed by a spin-coating step to fill the imprinted trench with core layer material. This waveguide cross section design smartly avoids critically controlling the thickness of the residual core layer. Figures 2(b) and 2(c) show the imprinted under-cladding trench and waveguide cross section after spin-coating the high refractive index layer, respectively.

\section{Experimental Results}

\subsection{Optical Transmission Response}

The PSQ-L polymer-based microring resonator can work at optical fiber communication bands, such as 1310 and $1550 \mathrm{~nm} .{ }^{15,16}$ The optical transmission spectrum of the fab- ricated microring resonator was measured by coupling light from a tunable laser (at $1550 \mathrm{~nm}$ band) to the waveguide via a lensed fiber. The transmitted light was also collected by a lensed fiber to the optical power meter. A polarization controller was used at the input port to select the polarization state of light. Figure 3 shows the measured transverse electric (TE) mode optical transmission spectrum of the microring resonator. The ER of $8 \mathrm{~dB}$ at the output port is obtained. The free spectrum range of the ring is about $0.582 \mathrm{~nm}$ as expected due to the large ring radius. The full-width at half-maximum (FWHM) is about $0.077 \mathrm{~nm}$. By taking the ratio between the resonant wavelength and FWHM, the Q-factor of about $2.0 \times 10^{4}$ is calculated. By fitting the experimental data with Eq. (1), $T$ and $A$ can be extracted as shown in Fig. 3(b). $T$ is about 0.58 , which means that $42 \%$ of the input optical power is coupled from the straight waveguide into the ring; $A$ is about 0.78 , which means $22 \%$ of the optical power is depleted after optical wave propagation per round-trip. Due to the optical loss of the waveguide microring resonator, the power of the resonant wavelength will be cut down, and it can be used as a notch filter. ${ }^{17}$

\subsection{Quasi-SSB Filtering Response}

Figure 4 shows the schematic diagram of the experiment setup for the polymer-based microring resonator used as a notch filter to realize the SSB radio over fiber transmission. An optical wave from the tunable laser source (TLS) is intensity-modulated by a radio-frequency (RF) signal generated from RF source (RFS). The output field is then applied to the polymer-based microring resonator. The erbium-doped fiber amplifier (EDFA) is used to compensate the coupling loss between the lensed fibers and the waveguides and the insertion loss of the polarization controller (PC). After transmitting over SMF, the optical property is measured by the

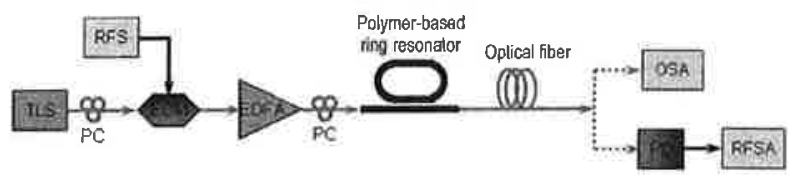

Fig. 4 Schematic diagram of the experiment selup. 


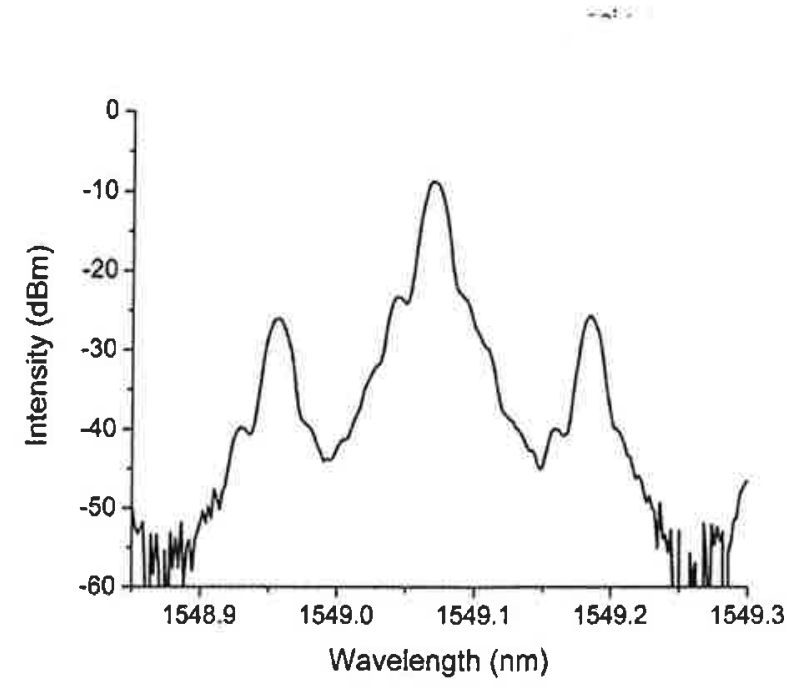

(a)

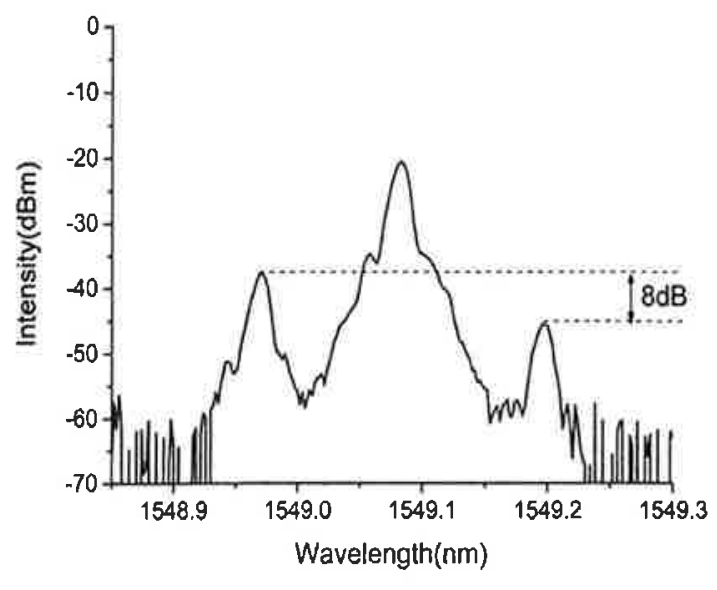

(b)

Fig. 5 Measured optical spectrums of the RF modulated optical wave (a) before and (b) after the polymer-based ring resonator.

optical spectrum analyzer (OSA) and the RF signal property from PD is measured by the RF spectrum analyzer (RFSA), respectively. Discrete EOM is used in this proof-of-principle demonstration, but it can eventually be integrated on the chip in the ultimate design. The polymer-based microring resonator serves as a notch filter to suppress one of the double sidebands (DSBs) of the microwave modulated optical wave. Then the SSB transmission over the SMF can be realized.

The sideband of the microwave modulated optical wave will be suppressed when it is located at the resonant wavelength of the polymer-based microring resonator. Figures 5(a) and 5(b) give the measured optical spectrums of the microwave $(14.35 \mathrm{GHz})$ modulated optical wave before and after the microring resonator. It can be seen that the right sideband is suppressed of about $8 \mathrm{~dB}$. The SSB filtering was not realized completely because the critical coupling condition ${ }^{17}$ was not satisfied. So the name of quasi-SSB filtering should be proper for the current polymer-based microring resonator.

The SSB filtering response can also be achieved by varying the optical wave frequency from the TLS when the frequency of the input microwave signal varies. In the experiment the optical wave from the TLS was selected to the proper frequency so that the right sideband frequency of the microwave modulated optical wave was placed at the resonant frequency of the polymer-based microring resonator. Figure 6(a)-6(d) show the quasi-SSB filtering response with microwave signal frequency from 10 to $22 \mathrm{GHz}$.

The suppression magnitude of the sideband is mainly decided by the ER of the waveguide microring resonator. Its variation in a certain range for different microwave frequency in Figs. 6(a)-6(d) was caused by the instability of the polymer-based microring resonator, which is an important issue to be taken into account. The optical notch response of the microring resonator could drift in wavelength due to changes in temperature since in polymer the refractive index depends on this parameter. Changes in optical power when high power signals are used also result in wavelength drifts. Many solutions are available in order to provide stability to the system, such as the athermal waveguide design, ${ }^{18}$ or dynamically controlling the refractive index of polymer by means of Kerr effect by applying an electrical field when the polymer has the electro-optical property. ${ }^{19}$ Improving the ring resonator design and its fabrication process would provide better Q-factor and higher ER.

\subsection{RF Carrying QPSK Signal Over Fiber Transmission}

Due to the chromatic dispersion of the SMF, the detected RF power of the DSB signal as shown in Fig. 4(a) suffers from the fading effect. ${ }^{20}$ The detected RF power will vary approximately $\mathrm{as}^{21}$

$P_{f_{R F}} \propto \cos \left(\frac{\pi L D \lambda_{C}^{2} f_{R F}^{2}}{c}\right)$,

where $D$ and $L$ are the dispersion coefficient and the length of the SMF, $\lambda_{\mathrm{C}}$ is the optical carrier wavelength, and $f_{\mathrm{RF}}$ is the microwave frequency. It attenuates as the chromatic dispersion accumulates over the long SMF and it vanishes when the product of the microwave frequency and the accumulated chromatic dispersion meets a certain value. However, the SSB signal transmission will avoid this chromatic dispersion induced fading effect, which can be realized by the fiber Bragg grating filter ${ }^{22}$ or the dual-electrode Mach-Zehnder modulator. $^{21}$

To demonstrate the validity of quasi-SSB filtering response of the polymer-based microring resonator, a QPSK signal of $20 \mathrm{Mbps}$ carried by $14.35 \mathrm{GHz}$ microwave was transmitted over $25 \mathrm{~km}$ SMF. For comparison, the DSB signal transmission was also tested, where the equal insertion loss of the integrated waveguide chip was provided by a tunable optical attenuator.

For the DSB signal transmission as shown in Fig. 7, the signal property deteriorated severely after the $25 \mathrm{~km}$ SMF with the error vector magnitude (EVM) from $4.6 \%$ to $54.4 \%$ and the signal-to-noise ratio (SNR) from 26.6 to $5.2 \mathrm{~dB}$. For the quasi-SSB one as shown in Fig. 8, the signal property remained well with the EVM from $5.3 \%$ to $9.9 \%$, the SNR from 25.5 to $20.6 \mathrm{~dB}$ before and after the $25 \mathrm{~km} \mathrm{SMF}$ transmission, respectively. Compared with DSB signal transmission, the quasi-SSB microwave carrying QPSK signal is transmitted over $25 \mathrm{~km}$ SMF successfully. 


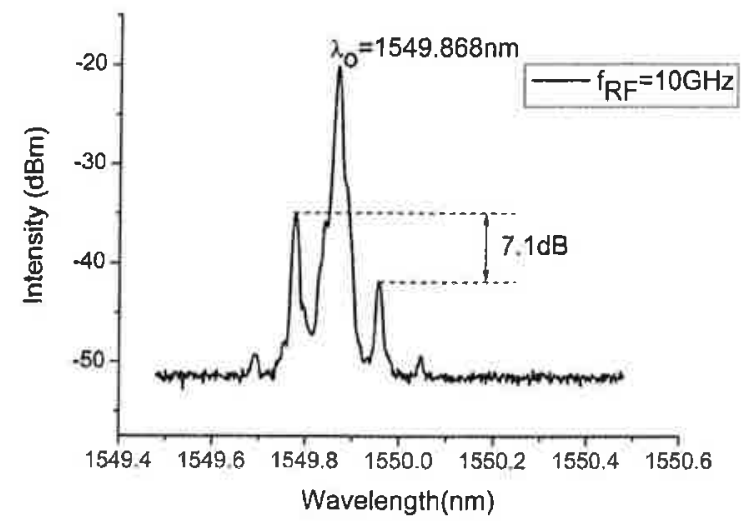

(a)

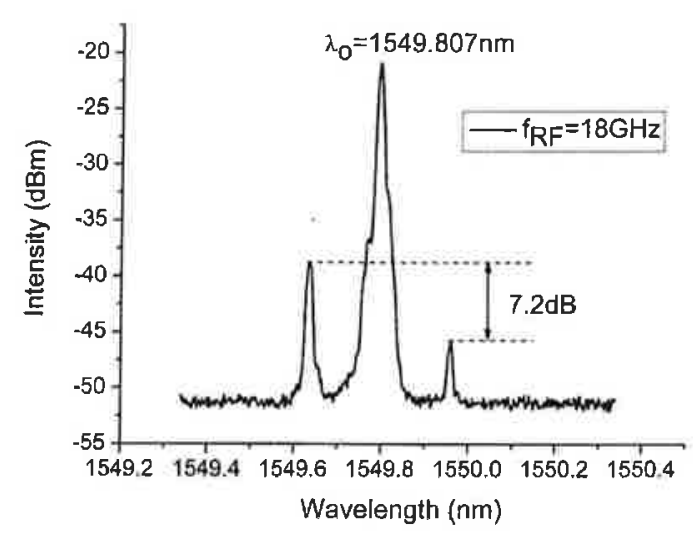

(c)

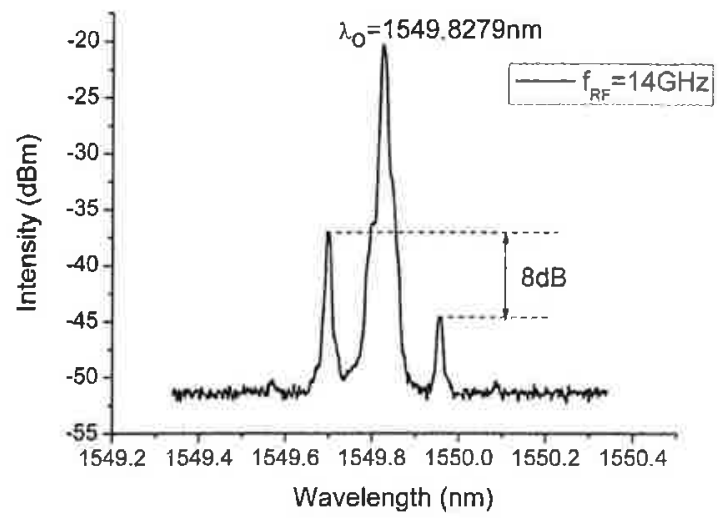

(b)

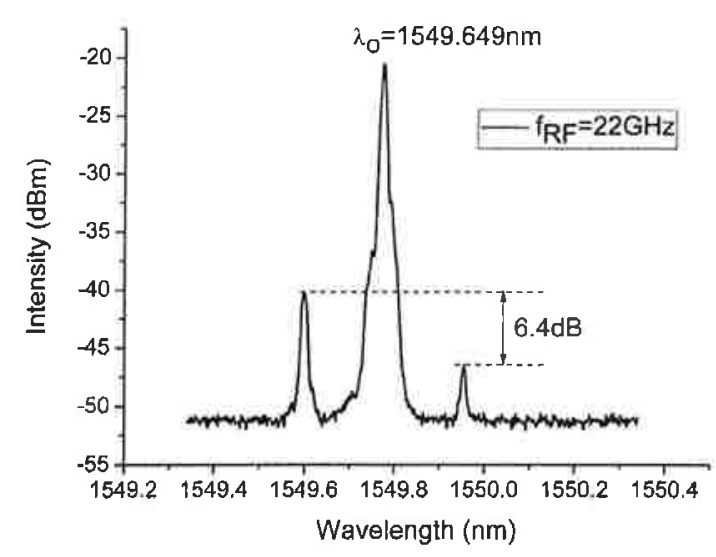

(d)

Fig. 6 Quasi-SSB filtering response of the polymer-based ring resonator with input microwave frequency of (a) $10 \mathrm{GHz}$, (b) $14 \mathrm{GHz}$ (c) $18 \mathrm{GHz}$ and (d) $22 \mathrm{GHz}$.

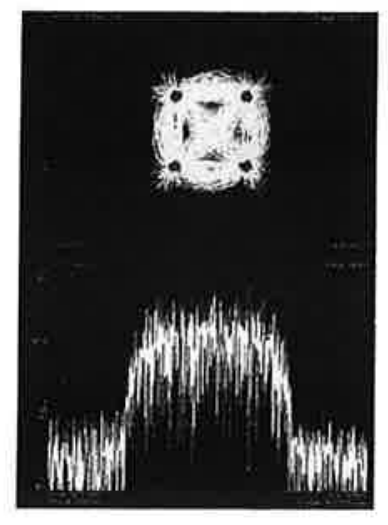

(a)

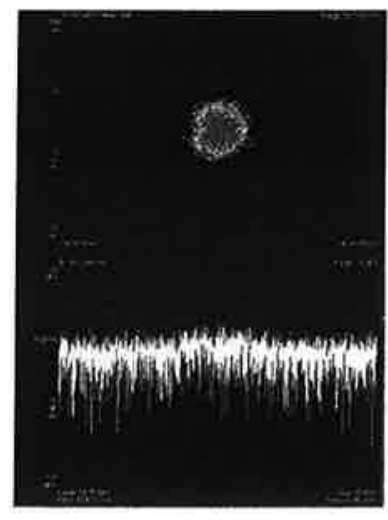

(b)

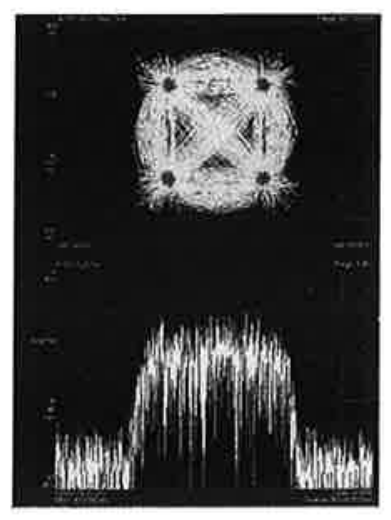

(a)

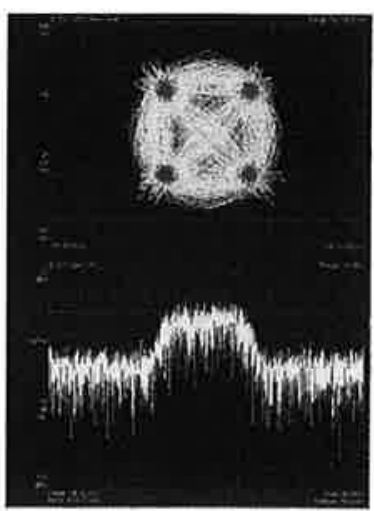

(b)

Fig. 7 DSB carrying QPSK signal transmission (a) belore and (b) after $25 \mathrm{~km}$ SMF.

Fig. 8 Quasi-SSB carrying QPSK signal transmission before (a) and after (b) $25 \mathrm{~km}$ SMF. 


\section{Conclusion}

In summary, an integrated waveguide microring resonator based on PSQ-L polymer was designed and fabricated by a simple UV-based soft-imprint technology. The quasi-SSB filtering response was achieved effectively by locating the sideband frequency of microwave modulated optical wave at the resonant frequency of the microring resonator. The chromatic dispersion caused fading effect was suppressed by the integrated waveguide filter with the demonstration of successful transmission of a $20 \mathrm{Mbps}$ QPSK signal carried by $14.35 \mathrm{GHz}$ microwave over $25 \mathrm{~km} \mathrm{SMF}$.

The ER of the microring resonator in the experiment was not very high since the critical coupling condition was not achieved properly. To improve the SSB filtering performance, further work of filter design and fabrication process should be carried out, such as cascading ring resonators, combination with Mach-Zehnder interferometer, and low waveguide loss.

\section{Acknowledgments}

This work was supported by the National Natural Science Foundation of China (Nos. 60807015, 61077015, and 60577014), Specialized Research Fund for the Doctoral Program of Higher Education (No. 200801411037), Natural Science Foundation of Liaoning Province (No. 20102020), and Fundamental Research Funds for the Central Universities (Nos. DUT10ZDG003 and DUT852006).

\section{References}

1. J. Capmany, B. Ortega, and D. Pastor, "A tutorial on microwave photonic filters," J. Lightwave Technol. 24(1), 201-229 (2006).

2. R. A. Minasian, "Photonic signal processing of microwave signals," IEEE Trans. Microwave Theory Tech. 54(2), 832-846 (2006).

3. J. Yao, "Microwave photonics," J. Lightwave Technol. 27(3), 314-335 (2009).

4. A. Seeds, F. Pozzi, C. C. Renaud, M. J. Fice, L. Ponnampalam, D. C. Rogers, L. F. Lealman and R. Gwilliam. "Microwave photonics: opportunities for photonic integration," 14th European Conference on Integrated Optics, Eindhoven, The Netherlands, pp. 123-132 (2008),

5. M. Y. Chen, X. Lu, H. Subbaraman, and R. T. Chen, "Fully printed phased-amay antenna for space communications," Proc SPIE 7318 $731814(2009)$

6. H. Schippers, J. Verpoorte, P. Joma, A. Hulzinga, A. Meijerink, C. G. H. Roeloffzen, L. Zhuang, D. A. I. Marpaung, W. van Etten, R. G. Heideman, A. Leinse, A. Borreman, M. Hoekman, and M. Wintels, "Broadband conformal phased array with optical beam forming for airborne satellite communication," IEEE Aerospace Conference, pp. 1-17 (2008).

7. L. Zhuang, C. G. H. Roeloffzen, A. Meijerink, M. Burla, D. A. I Marpaung, A. Leinse, M. Hoekman, R G. Heideman, and W. van Etten, "Novel ring resonator-based integrated photonic beamformer for Etten, "Novel ring resonator-based integrated photonic beamformer for
broadband phased array receive antennas-part II: experimental protobroadband phased array receive antennas-part II

8. L. Eldada, "The promise of polymers," SPIE's OE Magazine, pp, 26-29 (2002).

9. H. Ma, A. K.-Y. Jen, and L. R. Dalton, "Polymer-based optical waveguides: materials, processing, and devices," Adv. Mater: 14 (19), 1339 $1365(2002)$.

10. L. Eldada, "Advances in polymer-based dynamic photonic components, modules, and subsystems," Proc, SPIE 6351, 63510Y (2006).

11. B. Howley, X. Wang, M. Chen, and R. T. Chen, "Reconfigurable delay time polymer planar lightwave circuit for an $\mathrm{X}$-band phased-array antenna demonstration," J. Lightwave Technol. 25(3), 883-890 (2007).

12. C. Chen, Y. Yi, F. Wang, Y. Yan, X. Sun, and D. Zhang, "Ultra-long compact optical polymeric array waveguide true-time-delay line devices," IEEE J. Quantum Electron, 46(5), 754-761 (2010)

13. B. Bortnik, Y.-C. Hung, H. Tazawa, B.-J. Seo, J. Luo, A. K.-Y. Jen, W. H. Steier, and H. R. Fetterman, "Electrooptic polymer ring resonator modulation up to $165 \mathrm{GHz}$," IEEE J. Sel. Top. Quantum Electron. 13(1), 104-110 (2007)

14. G.-D. Kim and S.-S. Lee, "Photonic microwave reconfigurable filter based on a tunable polymeric ring resonator," Opt. Commun. 278, 303306 (2007).
15. H. B. Zhang, J. Y. Wang, L. K. Li, Y. Song, M. S. Zhao, and X. G Jian, "A study on liquid hybrid material for waveguides - Synthesi and property of PSQ-Ls for waveguides," J. Macromol. Sci. . Pure Appl Chem, 45, 232-237 (2008).

16. M. Zhao, X. Han, J. Teng, L. Wang, J. Wang, and X, Jian, "Recen progress on polymer materials and integrated optical waveguides," Proc. SPIE 7158, $71580 \mathrm{G}(2009)$

17. A. Yariv, "Universal relations for coupling of optical power between microresonators and dielectric waveguides," Electron. Left. 36(4), 321 $322(2000)$

18. X. Han, M. Zhao, J. Zhang, L. Wang, J. Teng, J. Wang, and X. Jian, "Design of athermal all-polymer waveguide microring resonator," Proc. SPIE 7630, 76301E (2009)

19. B.-J. Seo, S. Kim, B. Bortnik, H. Fetterman, D. Jin, and R. Dinu, "Optical signal processor using electro-optic polymer waveguides." J. Lightwave Technol. 27(15), 3092-3106 (2009).

20. U. Gliese, S. Ngrskov, and T.N. Nielsen, "Chromatic dispersion in fiberoptic microwave and millimeter-wave links," IEEE Trans. Microwave Theory Tech. 44(10), 1716-1724 (1996)

21. G. H. Smith, D. Novak, and Z. Ahmed, "Overcoming chromaticdispersion effects in fiber-wireless systems incorporating external modulators," IEEE Trans. Microwave Theory Tech. 45(8), 1410-1415 ulators,"

22. J. Park, W. V. Sorin and K. Y. Lau, "Elimination of the fibre chromatic dispersion penalty on $1550 \mathrm{~nm}$ millimetre-wave optical transmission," Electron. Lett. 33(6), 512-513 (1997).

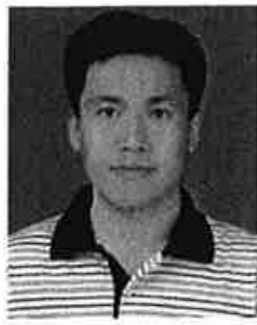

Xiuyou Han received his PhD degree in optical engineering from Shanghai Institute of Optics and Fine Mechanics, Chinese Academy of Sciences, Shanghai, China, in 2006. Currently, he is an associate professor in the School of Physics \& Optoelectronic Engineering, Dalian University of Technology, Dalian, China. His research interests are in the area of integrated photonic devices polymer photonics, and microwave photonics for optical communication and optical sensing systems.

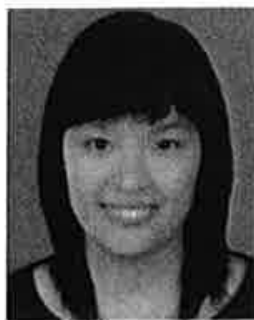

Jianing Zhang received her MS degree in optical engineering from Dalian University of Technology, Dalian, China, in 2011. Currently, she is an engineer in China United Network Communications Group Co., Ltd ("China Unicom"), Tianjin, China. Her MS dissertation was centered on the polymerbased waveguide devices for microwave photonic signal processing.

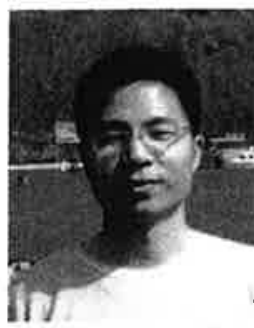

Linghua Wang received his BS degree in applied physics from Fuzhou University, Fuzhou, China, in 2006. He is currently working toward a PhD degree in the School of Physics \& Optoelectronic Engineering, Dalian University of Technology, Dalian, China, and since November 2009 he has been studying as a joint PhD student funded by the China Scholarship Council (CSC) in Ghent University, Ghent, Belgium. His research interests include design and fabrication of integrated photonic devices.

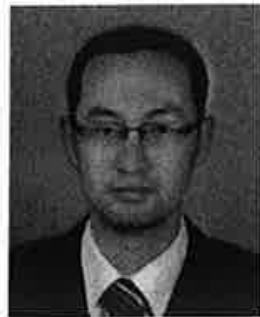

Yiying Gu received his PhD degree in optica engineering from Dalian University of Technology, Dalian, China, in 2011. Currently, he is working at Photonics Research Center School of Physics \& Optoelectronic Engineering, Dalian University of Technology. His current research fields are mainly in radio over fiber systems, integrated photonic devices for optical communications, and sensor systems. 


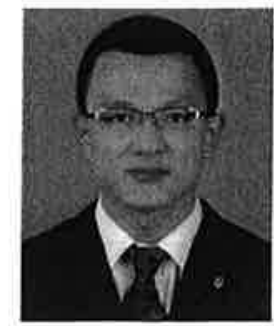

Meng Wang received his BS degree in optical information science and technology from Dalian University of Technology, Dalian, China, in 2010. He is pursuing his MS degree at the School of Physics \& Optoelectronic Engineering, Dalian University of Technology. His research fields are mainly in microwave photonics.

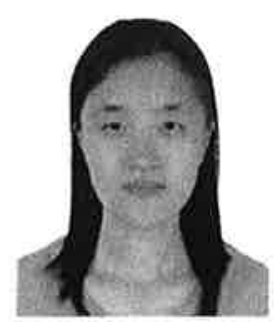

Jie Teng received her PhD degree in optical engineering from Dalian University of Technology, Dalian, China, in 2010. From 2008 to 2009, she studied as a joint PhD student funded by the China Scholarship Council (CSC) in Ghent University, Ghent, Belgium. Currently she is a lecturer in the school of Physics and Engineering, Henan University of Science and Technology, Luoyang, China. Her research interest mainly involves around polymer photonics.

Jinyan Wang: biography and photograph not available.

Xigao Jian: biography and photograph not available.

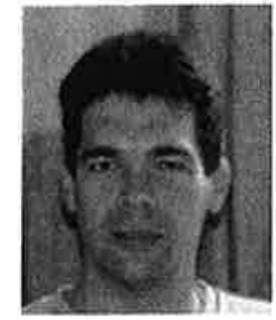

Geert Morthier received an MSc degree in electrical engineering and $\mathrm{a} P \mathrm{Ph}$ degree from Ghent University, Ghent, Belgium, in 1987 and 1991, respectively. Since 1991, he has been a member of Interuniversity Microelectronics Center, Ghent University, Ghent, Belgium, and since 2001, he is a part-time professor at Ghent University. His current research interests include the modeling and characterization of optoelectronic and photonic components, and different types of alloptical flip-flops for optical packet switching.

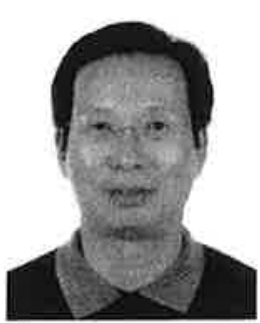

Mingshan Zhao received his PhD degree in electronic engineering from Ghent University, Belgium, in 2003. He is a professor in the School of Physics \& Optoelectronic Engineering, Dalian University of TechnologyDUT, Dalian, China. He leads the Photonics Research Center at DUT, which focuses on new concepts for microwave photonic components and systems, polymer-based photonic components, and circuits for optical communication and optical sensing. He has been a member of the technical program committees of International Topic Meeting on Microwave Photonics (AP-MWP'08 and MWP'09). 